\title{
Modeling Local Service Reliabilities for the Parcel Distribution System
}

\author{
Hyunwoo Lim*
}

\begin{abstract}
Demand for express parcel delivery has been greatly increasing in South Korea due to the growth of B2C e-commerce activities. It is imperative that parcel carriers have good insights into the performance of their distribution networks with different levels of demand and plan ahead for the adaptations in order to be able to meet future changes in demand. This paper proposes a framework to evaluate the likelihood of parcels arriving on-time to their destinations (local service reliability) with a strong focus being placed on their spatial distribution. The resulting maps of local service reliability allow us to identify specific locales that would suffer the most from a capacity overflow in the networking system, thus pointing to areas that need immediate attention. Furthermore, this paper attempts to identify potential factors which could affect the spatial variation of local service reliability.
\end{abstract}

Keywords: parcel delivery, service reliability, simulation, spatial analysis

Submission Date: 3/14/2011 Revision Date: 05/31/2011 Acceptance Date: 05/31/2011

* Professor, Graduate School of Logstics, Inha University, 253 Yonghyun-dong, Namgu, Incheon, Korea 402-751. Tel: +82-32-860-9234, Fax: +82-3-5841-8506, E-mail: hwlim@inha.ac.kr 


\section{Introduction}

The main goal of a logistics system is to move goods from an origin to a destination in a timely manner with the least possible cost. With the growth and proliferation of e-commerce activities, just-in-time production systems, and global production networks, small quantities of freight are frequently shipped over long distances (Anderson et al. 2003). Such requirements can impose great challenges to existing physical distribution networks, especially when there are great uncertainties in demand (Huppertz, 1999; Hesse, 2002). There fore, it is imperative for logistics service providers (LSP) to have good insights into the performance of their distribution networks with different levels of demand so that they can plan ahead for the adaptations that will need to take place in order to meet future changes in demand.

One of the most important performance measures in logistics systems is service reliability. It can be defined as the likelihood of successfully transporting the required amount of freight from an origin to a destination within a specified period of time (Iida, 1999). Understanding the potential changes of a logistics system's reliability under many possible situations allows practitioners to better prepare for uncertainties and contingent events such as natural disasters, traffic congestion, labor disputes, and major breakdowns of the system. Network reliability regarding connectivity, travel time, and capacity has been extensively studied in the telecommunications, power, and transportation networks (Wang et al.,2006). Many studies have focused on evaluating network reliability based on the probability of a link or node functioning. For instance, Lin (2006) proposed a reliability index as a probability that the maximum flow of the network equals the demand without exceeding the budget while taking into account each node's capacity. Researchers have studied the service reliability of logistics systems and have focused mostly on the supply reliability of supply chains for contingency logistics systems based on the reliability interference theory. Wang and Zhang (2003) conducted a quantitative analysis on the supply reliability of multi level supply chains based on the Markov process.

Typical retail distribution networks consist of hundreds or thousands of stores with multiple distribution centers (DC) where products are transported between retail stores and DC's. The service reliability of distribution networks depends on the spatial distribution of the DC's and the stores, the proximity of the stores to the DCs, transportation link connectivity, the capacities of the DCs, and the level of and delivery time requirements of the needed services. Wang et al.(2006) proposed reliability models for spatially distributed logistics networks based on the continuous approximation (CA) approach. Instead of modeling all of the network flows, they used store and demand densities as well as DC 
locations to estimate the service reliability at the system level and at the local distribution center level.

However, the reliability of parcel distribution networks has gained relatively less attention in comparison to supply chain networks. Furthermore, very few researches have investigated the spatial variation of reliabilities on partial distribution networks at the local level. Unlike all other logistics systems, parcel distribution networks must deal with small-sized frequent deliveries from individual customers who are scattered across large geographic areas. A typical parcel distribution system forms a hub-and-spoke network structure which includes local delivery centers (LDC), end-of-line (EOL) terminals, and one or more hubs. LDC's are responsible for collecting products from vendors (or shippers) and delivering packages to customers (or consignees). If the destination falls outside of the service area of the origin terminal, products must be transported to a hub for sorting and consolidation. Once packages are sorted at the hub, they are then sent to the EOL terminals near the destination and moved to the LDC to be delivered to each customer location.

Lim and Shiode (2011) investigated how cost efficiency and reliability of a physical distribution network can be affected by changes in parcel demand, and made recommendations regarding how they should respond to different types of changes. Using a discrete event simulation approach, possible adaptive measures regarding increased demand are tested at different levels of the decision making process within the parcel distribution network. The feasibility of the simulation was tested with parameters which had been adopted from the logistics service data of an existing major parcel carrier who operates in South Korea.

The service reliability of the parcel distribution networks depends on how well the LDCs are supported by the terminals, and the ability of the terminals and hubs to meet customer demands by processing packages in a timely manner. Modeling the reliability of such a system can also reveal the performance of the network under possible system disruptions such as exploding customer demand during the holiday seasons. Based on the simulation results from Lim and Shiode's (2011) research, this paper aims to further investigate how the spatial distribution of service reliabilities near destinations are affected by changes in parcel demand. It will then be possible to identify specific locales which could suffer the most from future capacity overflows in networks caused by potential demand increases. This will also indicate which terminals in the networks need immediate attention. Furthermore, this paper intends to investigate how reliability degradation can be affected by spatial distribution of parcel demand and travel time to the main hub. 


\section{Model Structure}

\subsection{Simulation of parcel distribution}

The parcel distribution network which was used in Lim and Shiode's (2011) research was based on actual information which was recorded by a South Korean express parcel delivery carrier. While the backbone structure of the original network was preserved, details were not replicated to protect proprietary business information. The resulting simplified network had the structure of a pure hub-and-spoke network consisting of one main hub terminal which was located in Daejeon, and ten EOL terminals which were located near major cities in each province. The origin-destination parcel flow was taken from the actual data and scaled down proportionately to the size of the network used in the simulation model. Please refer to their paper for any needed details regarding the simulation model including model assumptions and parameter specifications.

Table 1.

Testing four different scenarios

\begin{tabular}{l|c|c|c|c}
\hline & Scenario 1 & Scenario 2 & Scenario 3 & Scenario 4 \\
\hline $\begin{array}{l}\text { As-is: } \\
\text { Single hub with sorting } \\
\text { capacity of 4,000 parcels per } \\
\text { hour }\end{array}$ & $\sqrt{ }$ & $\sqrt{ }$ & & \\
\hline $\begin{array}{l}\text { Pre-sorting: } \\
\text { Assigning high priorities to } \\
\text { parcels heading to distant } \\
\text { terminals }\end{array}$ & & $\sqrt{ }$ & $\sqrt{ }$ & $\sqrt{ }$ \\
\hline $\begin{array}{l}\text { Introducing sub-hubs : } \\
\text { Delegate part of the sorting } \\
\text { process to regional hubs }\end{array}$ & & & $\sqrt{ }$ & \\
\hline $\begin{array}{l}\text { Main hub re-investment: } \\
\text { Single hub with sorting } \\
\text { capacity of } 6,000 \text { parcels per } \\
\text { hour }\end{array}$ & & & & \\
\hline
\end{tabular}

Source: Lim and Shiode (2011)

In order to explore the possible adaptive measures which could accommodate potential increases in parcel flow, Lim and Shiode (2011) performed simulations using four different scenarios as shown in Table 1. The first scenario serves as a base with which other 
scenarios can be compared and therefore represents the current condition of the system. It is based on the structure of a pure hub-and-spoke network where the single main hub operates with a sorting capacity of 4,000 parcels per hour. The second scenario represents a short-term adaptation at the operational level, which can be implemented on a daily basis to accommodate a temporary spike in the demand. During the sorting operation, it assigns a higher priority to parcels which are heading to remote terminals in order to help compensate for their relatively long travel times. If priority assignment is not enough to accommodate the increase in parcel flow, sub-hubs can be introduced as a tactical-level adjustment (Scenario 3). Finally, if the demand is expected to increase in the long run, a change at the strategic level can be made so as to increase the sorting capacity of the main hub to 6,000 parcels per hour.

In order to observe changes in the performance of the system with regards to the increasing parcel demand, ten different levels of parcel flows were considered in each scenario. Each of the subsequent levels of parcel flow was increased with the increment of $25 \%$ from the base level while the proportion of each OD flow remained constant. Given the stochastic nature of the travel time between terminals as well as the type of sorting operation at the hub, simulations were performed for 100 iterations at each level of parcel flow under the four different scenarios. The multiple iterations enabled us to show variations in the system performance measures and allowed us to derive statistically meaningful results. According to the simulation results from Lim and Shiode (2011), reliability problems due to delayed parcel arrivals were almost completely resolved in Scenarios 3 and 4. Therefore, this paper intends to analyze the local service reliability for Scenarios 1 and 2 .

\subsection{Local service reliabilities}

In general, travel time reliability $R_{i j}$ can be expressed as the probability of transporting parcels from origin $i$ to destination $j$ within a certain time $t_{0}$ (Iida, 1999).

$$
R_{i j}=P\left(T_{i j} \leq t_{0}\right)=1-P\left(T_{i j}>t_{o}\right)=1-P\left(V \leq d_{i j} / t_{o}\right)=1-F_{V}\left(d_{i j} / t_{o}\right)
$$

where

$T_{i j}$ is the travel time from $i$ to $j$;

$d_{i j}$ is the distance from $i$ to $j$; and

$F_{V}$ is the cumulative probability distribution function of travel speed $V$. 
In case parcels need to be sent to a hub for consolidation, travel time $T_{i j}$ should be considered as a combination of the travel time from the origin terminal $i$ to the hub $\left(T_{i h}\right)$, the processing time at hub $\left(T_{h}\right)$, and the travel time from the hub to the destination terminal $j\left(T_{h j}\right)$.

$$
T_{i j}=T_{i h}+T_{h}+T_{h j}
$$

Travel time reliability of all parcels heading to a particular terminal $j$ can be computed by finding the expected value of $R_{i j}$ However, this approach requires prior knowledge of the probability distribution of the processing time at the hub and the travel time between each EOL terminal and the hub. Instead of using this method, this paper estimates the local terminal reliability from the simulation results by obtaining the proportion of parcels which were successfully transported to a specific EOL terminal within a specified time (12 hours) taken from departing the origin terminal. Then, the local service reliability at terminal $j$ $\left(R_{j}\right)$ can be simply calculated as such:

$$
R_{j}=1-\frac{\Sigma_{i} f_{i j}}{\sum_{i} D_{i j}}
$$

where

$D_{i j}$ Number of parcels to be transported from terminal $i$ to $j$;

$f_{i j} \quad$ Number of parcels transported from terminal $i$ to $j$ after the cut-off time

Since the simulation of the parcel distribution network was performed 100 times for each level of the parcel demand under each scenario, the average local terminal reliability was taken based on the analysis. Once the local service reliabilities were obtained for the ten EOL terminals, they were then used in the simulation model, they were then spatially interpolated across the entire country in order to be visualized on a map. Inverse distance weighting (IDW) is one of the widely used spatial interpolation methods that estimates the unknown value of location $i$ by taking the weighted average of the known values of the points around it (O'Sullivan and Unwin, 2010). Weights are assumed to be inversely proportionate to the power of distance between estimation points $i$ and the surrounding points( $j$ 's). These will be used for interpolation and the larger weights will be assigned to points closer to $i$. The basic idea behind this is Tobler (1970)'s First Law of Geography : "Everything is related to everything else, but near things are more related than distant things." 


$$
R_{i}=\sum_{j \in N} \frac{1 / d_{i j}^{k}}{\sum_{j \in N} 1 / d_{i j}^{k}} R_{j}
$$

where

$R_{i}$ is the unknown local service reliability at $i$ to be estimated;

$R_{j}$ is the known local service reliability at location $j$ around $i$;

$N$ is a set of points to be used for interpolation

$k>0$

Comparing the maps of local service reliabilities for different levels of parcel demand allows us to understand how reliabilities will vary across spaces when we have a change in demand. Furthermore, we must attempt to identify factors which will affect the variation of local service reliabilities by using multiple linear regression models. Spatially interpolated reliability values can be assigned to the entire 67 EOL terminals which are operated by the company being studied. The reliability values for each terminal $\left(R_{j}\right)$ can then be regressed by two explanatory variables: average travel time between the hub and terminal $\left(T_{h j}\right)$ and the parcel demand at the terminal $\left(D_{j}\right)$. Since all parcels are assumed to be consolidated and sorted at a single hub, parcel delays due to congestion at the hub will not show any spatial variations and will be captured by the constant $\beta_{o}$ in the regression model.

$$
R_{j}=\beta_{o}+\beta_{1} T_{h j}+\beta_{2} D_{j}
$$

Once the explanatory variables are confirmed to be statistically significant and the regression residuals show signs of spatial patterns, Geographic Weighted Regression analysis (GWR) can be performed in order to explain the spatial variations in the regression coefficients. GWR creates a regression coefficient surface for each explanatory variable. As shown in equation (6), GWR creates a regression equation for each observation in the study region. The resulting GWR coefficient surfaces illustrate how relationships between the dependent variable and each explanatory variables will vary within the designated space (Fotheringham et al.2002).

$$
R_{j}=\beta_{o j}+\beta_{1} T_{h j}+\beta_{2 j} D_{j}, j=1,2,, \mathrm{n}
$$




\section{RESULTS}

\subsection{Local service reliabilities under the As-Is scenario (Scenario 1)}

Table 2.

Average local terminal reliabilities from Scenario 1

\begin{tabular}{c|c|c|c|c|c|c|c|c|c|c}
\hline $\begin{array}{c}\text { Demand } \\
\text { level }\end{array}$ & $\begin{array}{c}1 \\
\text { Bundang }\end{array}$ & $\begin{array}{c}2 \\
\text { Bugok }\end{array}$ & $\begin{array}{c}3 \\
\text { Hanam }\end{array}$ & $\begin{array}{c}4 \\
\text { Daegu }\end{array}$ & $\begin{array}{c}5 \\
\text { Pohang }\end{array}$ & $\begin{array}{c}6 \\
\text { Yangsan }\end{array}$ & $\begin{array}{c}7 \\
\text { Gwangju }\end{array}$ & $\begin{array}{c}8 \\
\text { Jeonju }\end{array}$ & $\begin{array}{c}9 \\
\text { Gangrung }\end{array}$ & $\begin{array}{c}10 \\
\text { Sokcho }\end{array}$ \\
\hline \hline 2 & 1.00 & 1.00 & 1.00 & 1.00 & 1.00 & 1.00 & 1.00 & 1.00 & 1.00 & 1.00 \\
\hline 3 & 1.00 & 1.00 & 1.00 & 1.00 & 1.00 & 1.00 & 1.00 & 1.00 & 1.00 & 1.00 \\
\hline 4 & 1.00 & 1.00 & 1.00 & 1.00 & 1.00 & 0.78 & 1.00 & 1.00 & 0.43 & 0.74 \\
\hline 5 & 0.89 & 0.99 & 0.94 & 0.84 & 1.00 & 0.82 & 0.85 & 1.00 & 0.43 & 0.62 \\
\hline 6 & 0.73 & 0.66 & 0.63 & 0.74 & 0.95 & 0.69 & 0.93 & 1.00 & 0.39 & 0.42 \\
\hline 7 & 0.62 & 0.49 & 0.44 & 0.66 & 0.81 & 0.65 & 0.83 & 0.67 & 0.37 & 0.24 \\
\hline 8 & 0.58 & 0.43 & 0.36 & 0.54 & 0.52 & 0.53 & 0.77 & 0.79 & 0.28 & 0.33 \\
\hline 9 & 0.52 & 0.46 & 0.30 & 0.61 & 0.54 & 0.54 & 0.69 & 0.65 & 0.29 & 0.16 \\
\hline 10 & 0.48 & 0.40 & 0.26 & 0.54 & 0.43 & 0.50 & 0.63 & 0.55 & 0.24 & 0.14 \\
\hline
\end{tabular}




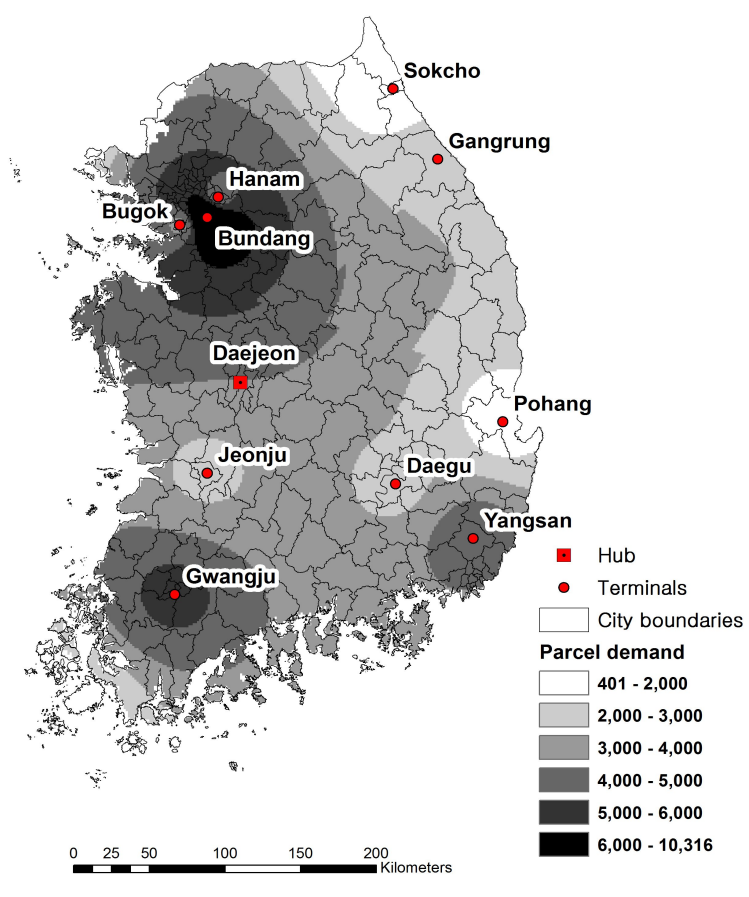

Figure 1.

Interpolated surface of parcel demand (Level 6)

One of the objectives of this paper is to find out how local service reliabilities across the country change when consistent parcel demand increases over time. Assuming an 18\% annual growth rate, parcel demand will increase 2.25 times from the base year level in a five year period. While the simulation was performed on ten levels of parcel demand, this paper focuses on the local service reliabilities at Level 6 which correspond to the demand increase in the next five years. Figure 1 shows the amount of parcel demand at level 6 interpolated from the values for the ten EOL terminals used in the simulation model. Note that the demand is heavily concentrated around the Seoul Metropolitan Area and much less concentrated in the peripheral regions such as Gangwon Province.

Table 2 shows the average local terminal reliability at each destination terminal for the base scenario. When overall system reliability began to drop below one in Level 3, problems with late parcels began to appear first in Yangsan (Terminal 6), Gangrung (Terminal 9) and Sokcho (Terminal 10), which are located relatively far from the hub requiring 4 - 5 hours of travel time. Sokcho (Terminal 10) does not show as much reliability degradation even though it is located just about the same distance from the hub as Gangrung (Terminal 9). This is because it only demands 400 units of parcels which require 
only a single trip by a line-haul truck. Terminal reliability starts to drop below one as the total parcel flow increases. The reliability of Bundang (Terminal 1), Bugok (Terminal 2), and Hanam (Terminal 3) which are located in the Seoul Metropolitan Area also drops significantly at Level 5 and beyond.

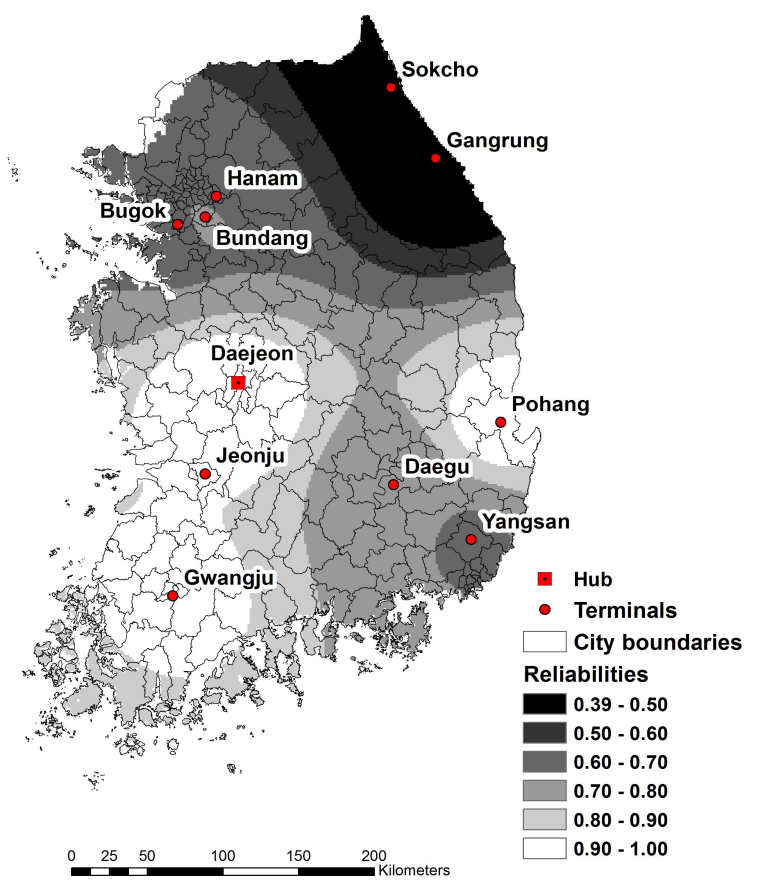

Figure 2.

Interpolated surface of local service reliability (Scenario 1: Level 6)

Figure 2 shows the continuous surface of the local reliabilities which were estimated using the reliability measures of the 10 terminals using the inverse distance weighted (IDW) spatial interpolator. If the exact logistics boundaries of each terminal were known, a choropleth map would be the best way to visualize the local reliability for all of South Korea. However, without such information available, spatial interpolation provides reasonable estimates of local reliabilities throughout the country. It should be noted that the estimates may vary slightly by using the spatial interpolation methods and their parameters. In Level 6, local reliabilities in Gangwon Province are as low as 40\% while those around the Seoul Metropolitan Areas are below 60\%. On the other hand, the areas around Gwangju (Terminal 7) and Jeonju (Terminal 8) have both maintained reliability above $90 \%$. 


\section{Table 3.}

OLS regression results (Scenario 1: Level 6)

\begin{tabular}{|c|c|c|c|}
\hline \multicolumn{3}{|c|}{$\begin{array}{l}\text { Number of observations: } 67 \\
\text { R-square }=0.2529 \\
\text { F-statistic }=10.8341 \quad(p \text {-value }=0.00) \\
\text { Moran's I for } \quad \text { standard residual }=0.17 \text { (p-value }=\end{array}$} & \multirow{2}{*}{$\begin{array}{l}\text { 00) } \\
\text { p-value }\end{array}$} \\
\hline & Coefficient & t-statistic & \\
\hline Intercept & 0.9871 & 15.6034 & 0.00 \\
\hline Travel time to hub & -0.0732 & -3.86 & 0.00 \\
\hline Parcel demand & -0.000021 & -2.31 & 0.02 \\
\hline
\end{tabular}

According to the OLS regression results, the travel time to the hub has a significantly negative relationship with regards to local service reliability (Table 3 ). This explains the extremely low reliability rating in Gangwon Province, which is a remote terminal located far away from the main hub in Daejeon. However, travel time to the hubs does not solely explain the local service reliability variations throughout the country. For instance, the Gwangju area shows a much higher reliability than the Seoul Metropolitan Area even though both areas are an equal distance away from the hub. A relatively low level of reliability in the Seoul Metropolitan Area can be explained by the large concentration of parcel demand. This can be supported by the regression coefficient for parcel demand which turns out to have a negative statistical significance even though its absolute quantity is relatively small. The intercept in the OLS regression can be considered as a constant effect to the local service reliability at certain levels in parcel demand such as congestion in the hub. Moran's I statistic for the standard residual suggests that there is a positive spatial autocorrelation (strong spatial pattern) in the regression residual. 


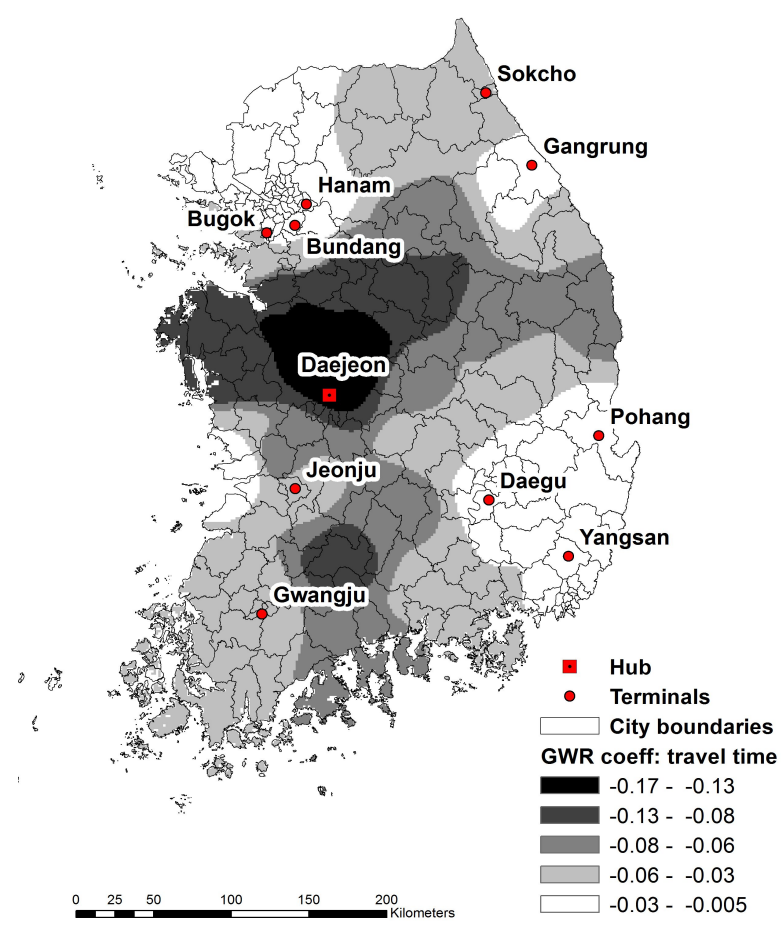

Figure 3.

GWR coefficient surface of travel time to the hub (Scenario 1: Level 6)

GWR results clearly indicate that the regression coefficient for travel time to the hub significantly varies over long distances (Figure 3). While the sign of the coefficient is dominantly negative as predicted in the OLS regression, strong negative values are concentrated around Daejeon. This implies that the local service reliabilities are more sensitive to marginal changes in travel time to the hub in those areas.

\subsection{Local service reliabilities under the Pre-Sorting scenario (Scenario 2)}

Since it was determined that terminals which are remotely located from the hub end up having the most parcel delays, it would be reasonable to assign higher priorities to parcels heading to those areas. Parcels which are assigned higher priorities are processed first in the queue during the sorting process in the hub when contending with parcels which were assigned lower priorities. This can serve as one of the immediate attempts to improve the reliability of the system when facing increasing demand, and it has also been shown to improve the overall system reliability according to the simulation results (Lim and Shiode 2011). 


\section{Table 4.}

Average local reliabilities from Scenario 2

\begin{tabular}{c|c|c|c|c|c|c|c|c|c|c}
\hline & $\begin{array}{c}1 \\
\text { Bundang }\end{array}$ & $\begin{array}{c}2 \\
\text { Bugok }\end{array}$ & $\begin{array}{c}3 \\
\text { Hanam }\end{array}$ & $\begin{array}{c}4 \\
\text { Daegu }\end{array}$ & $\begin{array}{c}5 \\
\text { Pohang }\end{array}$ & $\begin{array}{c}6 \\
\text { Yangsan }\end{array}$ & $\begin{array}{c}7 \\
\text { Gwangju }\end{array}$ & $\begin{array}{c}8 \\
\text { Jeonju }\end{array}$ & $\begin{array}{c}9 \\
\text { Gangrung }\end{array}$ & $\begin{array}{c}10 \\
\text { Sokcho }\end{array}$ \\
\hline \hline 1 & 1.00 & 1.00 & 1.00 & 1.00 & 1.00 & 1.00 & 1.00 & 1.00 & 1.00 & 1.00 \\
\hline 2 & 1.00 & 1.00 & 1.00 & 1.00 & 1.00 & 1.00 & 1.00 & 1.00 & 1.00 & 1.00 \\
\hline 3 & 1.00 & 1.00 & 1.00 & 1.00 & 1.00 & 1.00 & 1.00 & 1.00 & 1.00 & 1.00 \\
\hline 4 & 1.00 & 1.00 & 1.00 & 1.00 & 1.00 & 1.00 & 1.00 & 1.00 & 1.00 & 1.00 \\
\hline 5 & 0.98 & 1.00 & 0.83 & 0.83 & 1.00 & 1.00 & 0.83 & 1.00 & 1.00 & 1.00 \\
\hline 6 & 0.68 & 0.56 & 0.74 & 0.74 & 1.00 & 1.00 & 0.93 & 1.00 & 1.00 & 1.00 \\
\hline 7 & 0.70 & 0.50 & 0.44 & 0.67 & 1.00 & 1.00 & 0.83 & 0.33 & 1.00 & 1.00 \\
\hline 8 & 0.47 & 0.46 & 0.40 & 0.60 & 1.00 & 1.00 & 0.61 & 0.60 & 1.00 & 1.00 \\
\hline 9 & 0.51 & 0.21 & 0.37 & 0.56 & 1.00 & 1.00 & 0.56 & 0.56 & 1.00 & 1.00 \\
\hline 10 & 0.54 & 0.00 & 0.34 & 0.51 & 1.00 & 1.00 & 0.51 & 0.26 & 1.00 & 1.00 \\
\hline
\end{tabular}

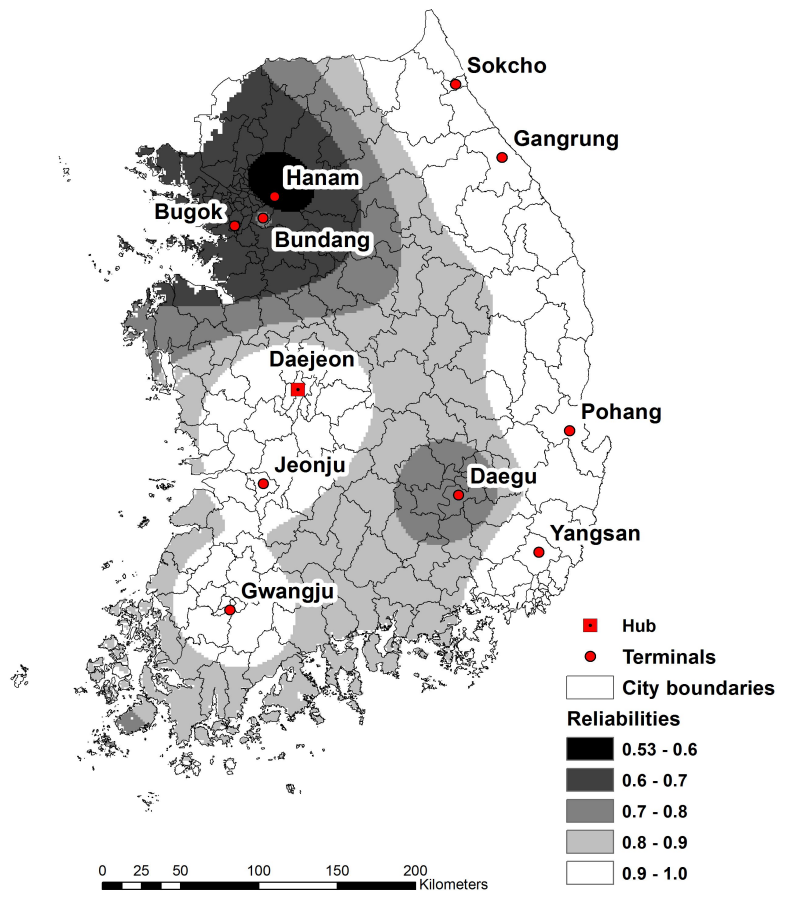

Figure 4.

Interpolated surface of local service reliability (Scenario 2: Level 6) 
As shown in Table 4, remotely located terminals which are assigned higher priorities such as Pohang (Terminal 5), Yangsan (Terminal 6), Gangrung (Terminal 9), and Sokcho (Terminal 10) continue to have a high level of reliability through all ten levels of parcel demand. On the other hand, the rest of the terminals still have problems with parcel delays when assigning priorities is not considered. Bundang (Terminal 1), Bugok (Terminal 2), and Hanam (Terminal 3) in particular experience more delays compared to the base scenario. This is possibly due to the relatively large parcel volume. Under extreme circumstances, none of the parcels arrive on time in Bogok (Terminal 2) under Level 10 circumstances. Local service reliability maps for Level 6 (Figure 4) indicate that most areas on the East coast maintain almost $100 \%$ reliability, while less than $70 \%$ of the parcels arrive on-time in the areas located around the Seoul Metropolitan Area. This indicates that priority assignment in the sorting process is not the ultimate solution to prevent parcel delay problems when the demand exceeds the limited sorting capacity in a hub.

\section{Table 5.}

OLS regression results (Scenario 2: Level 6)

\begin{tabular}{|l|l|l|l|}
\hline $\begin{array}{l}\text { R-square }=0.4168 \\
\text { F-statistic }=22.8730 \quad(p-v a l u e=0.00) \\
\text { Moran's I for standard residual }=0.59 \quad(p \text {-value }=0.00)\end{array}$ \\
\hline & Coefficient & t-statistic & p-value \\
\hline Intercept & 0.9448 & 13.20 & 0.00 \\
\hline Travel time to hub & 0.0352 & 1.91 & 0.06 \\
\hline Parcel demand & -0.000058 & -5.78 & 0.00 \\
\hline
\end{tabular}

When performing OLS regression on the local service reliability under Scenario 2 at Level 6, the regression coefficient for travel time to a hub turns out to have a positive sign in contrast to the case in Scenario 1. This implies that service reliabilities are improved for terminals which are distant from the hub due to assigning higher sorting priorities heading to those areas. The regression coefficient for parcel demand was determined to be slightly more negative with statistical significance compared to that of Scenario 1. Moran's I statistic of 0.59 implies that there is a strong spatial autocorrelation (spatial pattern) in the regression residuals. This suggests that either another explanatory variable should be added to the model or that a spatial regression such as GWR must be performed. 


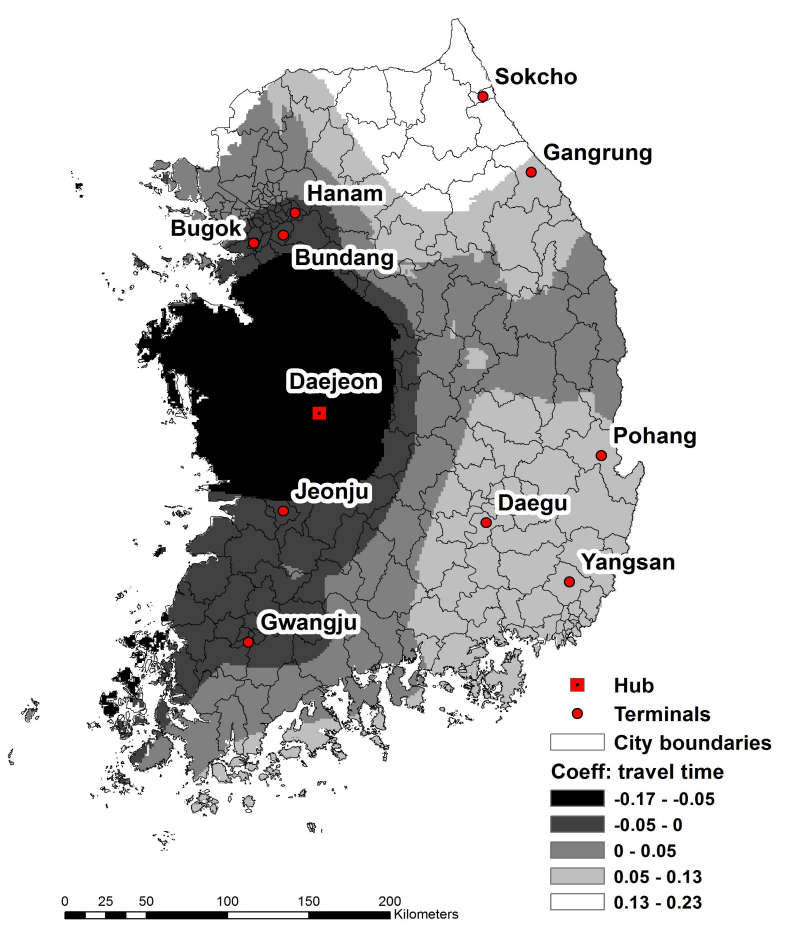

Figure 5.

GWR coefficient surface of travel time to the hub (Scenario 2: Level 6)

Figure 5 illustrates the GWR coefficient surface for travel time to the hub. Interestingly, not only the absolute magnitude, but the sign of the regression coefficient turned out to vary significantly over long distances. Coefficients were predominantly positive in Gangwon Province which implies that local service reliabilities are relatively higher in areas which are located farther away from the hub. Since the local service reliability in areas which are far from the hub can be improved by using the priority assignment policy, there is a positive relationship between the travel time to the hub and the local reliability in those areas. On the other hand, the regression coefficients show negative signs in the western part of the country, especially in the Daejeon area. This implies that the terminal's proximity to the hub is somewhat more important in those areas in terms of maintaining a certain level of service reliability. 


\section{CONCLUSIONS}

The demand for express parcel delivery has been constantly increasing in South Korea due to the growth of $\mathrm{B} 2 \mathrm{C}$ e-commerce activities. Accommodating such an increase in demand has always been a great challenge for parcel carriers. When parcel flow exceeds the sorting capacity of the hub, congestion in the hub causes delays in parcel delivery resulting in reliability degradation throughout the entire system. The unique contribution of this study is that it suggests a framework to evaluate the spatial distribution of local service reliability throughout the parcel distribution system. The likelihood of parcels arriving on-time are estimated for a selected number of terminals at different levels of parcel demand through the simulation, and spatially interpolated across all of South Korea.

The resulting maps which show local service reliability identify specific locales which would suffer the most in the event of a capacity overflow in the network, thus pointing out areas which need immediate attention. The OLS regression model identified travel time to the hub facilities and parcel demand as the main factors which decrease the chances of on-time delivery. According to the case study which was performed on a single parcel carrier in South Korea, Gangwon Province had the greatest amount of parcel delay problem because of its remote location far away from the hub in Daejeon. Significant spatial variation was also found in the strength of the relationship between the proximity of the hub and the local reliability when performing GWR. The Seoul Metropolitan Area also suffers from parcel delays due to the large demand concentration in the event of capacity overflows in the hubs.

In order to make immediate attempts to mitigate parcel delay problems due to increases in demand, assigning higher sorting priorities in the hubs to parcels which are heading to remote locations turned out to be an effective way to reduce parcel delays across the country. These findings can help the logistics service providers make an educated decision when they need to accommodate higher demand in an attempt to provide on-time deliveries in order to improve their level of customer service. 


\section{Acknowledgements}

I would like to thank Inha university for supporting this research through the Research Grant (INHA-42160), I also thank the referees and the associated editor for valuable suggestions.

\section{References}

Anderson, W. P., Chatterjee, L. and Lakshmanan, T. R. (2003) E-commerce, transportation, and economic geography, Growth and Change, 34,415-432.

Fotheringham, A. S., Brundson, C., and Charlton, M. (2002) Geographically Weighted Regression: The Analysis of Spatially Varying Relationships, Wiley.

Huppertz, P. (1999) Market changes require new supply chain thinking, Transportation and Distribution, 40(3),70-74.

Iida, Y. (1999) Basic concepts and future directions of road network reliability analysis, Journal of Advanced Transportation, 33,125-134.

Hesse, M. (2002) Shipping news: the implications of electronic commerce for logistics and freight transport, Resources, Conservation, and Recycling, 36,211-240.

Lim, H. and Shiode, N. (2011) The impact of online shopping demand on physical distribution networks: a simulation approach, International Journal of Physical Distribution and Logistics Management, forthcoming.

Lin, Y.-K. (2006) Evaluate the performance of a stochastic-flow network with cost attribute in terms of minimal cuts, Reliability Engineering and System Safety, Vol.91, 539-545.

O'Sullivan, D. and Unwin, D. (2010)Geographic Information Analysis, $2^{\text {nd }}$ Ed,Wiley.

Thomas, M. U. (2002) Supply chain reliability for contingency operations, in Proceedings Annual Reliability and Maintainability Symposium, January 2002, 61-67.

Tobler W. (1970) A computer movie simulating urban growth in the Detroit region, Economic Geography,46(2):234-240.

Wang, J. and Zhang, W. J. (2003) Reliability analysis of supply chain system, China Safety Science Journal, 11, 73-75.

Wang, N. and Lu, J.-C., Kvam, P. (2006) Reliability modeling in spatially distributed logistics systems, IEEE Transactions on Reliability, 55(3),525-534.

Yi, C. and Ju, S. (2006) Service reliability analysis on logistics network, in Proceedings of IEEE International Conferences on Service Operations and Logistics, and Informatics, Shanghai, 21-23June2006. 
